\title{
Selective cognitive empathy deficit in adolescents with restrictive anorexia nervosa
}

\author{
This article was published in the following Dove Press journal: \\ Neuropsychiatric Disease and Treatment \\ 16 October 2013 \\ Number of times this article has been viewed
}

\section{Sara Calderoni' \\ Pamela Fantozzi' \\ Sandra Maestro' \\ Elena Brunori' \\ Antonio Narzisi' \\ Giulia Balboni² \\ Filippo Muratori ${ }^{1,3}$ \\ 'Department of Child Neurology and Psychiatry, IRCCS Stella Maris \\ Foundation, ${ }^{2}$ Department of Surgery, Medical, Molecular and Critical \\ Area Pathology, University of Pisa, \\ ${ }^{3}$ Department of Developmental \\ Medicine, University of Pisa, Pisa, Italy}

Background: A growing, but conflicting body of literature suggests altered empathic abilities in subjects with anorexia nervosa-restricting type (AN-R). This study aims to characterize the cognitive and affective empathic profiles of adolescents with purely AN-R.

Methods: As part of a standardized clinical and research protocol, the Interpersonal Reactivity Index (IRI), a valid and reliable self-reported instrument to measure empathy, was administered to 32 female adolescents with AN-R and in 41 healthy controls (HC) comparisons, matched for age and gender. Correlational analyses were performed to evaluate the links between empathy scores and psychopathological measures.

Results: Patients scored significantly lower than HC on cognitive empathy (CE), while they did not differ from controls on affective empathy (AE). The deficit in CE was not related to either disease severity nor was it related to associated psychopathology.

Conclusion: These results, albeit preliminary, suggest that a dysfunctional pattern of $\mathrm{CE}$ capacity may be a stable trait of AN-R that should be taken into account not only for the clinical management, but also in preventive and therapeutic intervention.

Keywords: anorexia nervosa-restricting type, cognitive empathy, affective empathy, female adolescents, Interpersonal Reactivity Index

\section{Introduction}

Anorexia nervosa (AN) is a serious psychiatric disorder affecting mainly young females and it is associated with poor long-term outcome. ${ }^{1}$ According to the current version of the Diagnostic and Statistical Manual of Mental Disorders, the essential features of AN are a significantly low body weight for the developmental stage of the subject, an intense fear of gaining weight or persistent behavior that interferes with weight gain, and disturbance in experiencing body weight and shape. ${ }^{2}$ Two different subtypes of the disorders are specified, the restricting type (AN-R), in which weight loss is accomplished exclusively through a massive reduction in food intake and excessive exercise over the previous 3 months, and the binge-eating/purging type (AN-BE), in which binge eating is usually associated with purging through self-induced vomiting or misuse of laxatives and diuretics or enemas during the previous 3 months. ${ }^{2}$ Despite neither diagnostic criteria nor reports on common associated psychopathology describe empathic alterations in AN, clinical observation as well as some research data suggests that empathic difficulty could be part of the AN phenotype. ${ }^{3}$ Empathy is a complex multidimensional construct and a core component of social cognition. A schematic distinction of empathy includes: a) sharing the emotional states of others (affective empathy $[\mathrm{AE}]$ ), and $\mathrm{b}$ ) understanding the perspective of another person (cognitive
Correspondence: Filippo Muratori IRCCS Stella Maris Foundation, Via dei Giacinti, 2, I-560I8 Calambrone, Pisa, Italy

Tel +39050886323

Fax +39050886247

Email filippo.muratori@inpe.unipi.it 
empathy $[\mathrm{CE}]){ }^{4}$ A phenomenon partially overlapping with empathy is theory of mind (ToM). ToM consists of the ability to make inferences regarding others' emotions (affective or emotional ToM) or beliefs and motivations (cognitive ToM); while the latter is strongly similar to CE, the former may be related to both affective and cognitive empathy. ${ }^{5}$

A considerable subgroup of AN subjects with associated empathy disorders was revealed in a Gillberg et al study: ${ }^{6}$ the authors described these patients as impaired in understanding the cognitive and emotional perspectives of other people and with a clinical picture suggestive of an autism spectrum disorder (ASD). Subsequent reports confirmed autistic traits as predictive ${ }^{1}$ or comorbid factors in AN patients: ${ }^{7}$ this has led some authors to consider AN as a version of ASD. ${ }^{8}$ However, an impaired empathic profile in AN is not a consistent finding; for example, a self-report evaluation of empathic abilities failed to detect statistically significant differences between AN patients and healthy controls. ${ }^{9}$ On the other hand, Tchanturia et $\mathrm{l}^{10}$ first explored cognitive ToM in women with AN, revealing a clear impairment in a subgroup of these patients of which the study lacked the power to demonstrate statistically. Later reports showed abnormalities in both affective and cognitive ToM, ${ }^{11}$ in emotional ToM only, ${ }^{12}$ or no relevant differences between adult patients with AN and controls in emotional ToM. ${ }^{13}$ Finally, a recent investigation that evaluated, among other things, the social perception domain through a multidomain neuropsychological battery designed specifically for children and adolescents, reported an absence of deficit in this area in AN-R patients. ${ }^{14}$

The assessment of empathic response can be performed through physiological measurements (eg, heart rate or skin conductance registration), indirect indices (facial, gestural, bodily, and vocal expression recognition), and psychological evaluation (test administration, reports from significant others, and self-reported questionnaires). Among self-reported psychological tests aimed at evaluating empathic functioning, the Interpersonal Reactivity Index (IRI) represents one of the most widely used and comprehensive instruments. ${ }^{4}$ In fact, different abnormalities in CE and AE levels, assessed through the IRI, have been reported in a variety of psychiatric conditions, including psychopathy, ${ }^{15}$ borderline personality disorder, ${ }^{16}$ Asperger syndrome, ${ }^{17}$ schizophrenia, ${ }^{18}$ major depressive disorder, ${ }^{19}$ bipolar disorder, ${ }^{20}$ and patients with alcohol dependence. ${ }^{21}$ To our knowledge, no study has evaluated empathy abnormalities in adolescents with AN-R.

In this study, we analyzed the empathic abilities of a homogeneous group of adolescents with AN-R. As AN-R subjects are characterized by starvation and associated internalizing psychopathology that can impact cognitive abilities, ${ }^{22}$ we specifically investigated the empathic profile controlling for disease severity and associated psychopathology.

\section{Materials and methods \\ Participants}

Thirty-two adolescent females (mean age \pm standard deviation $[\mathrm{SD}]=14.78 \pm 1.75$ years; range $=11.00-17.33$ years) with AN-R were consecutively recruited from the inpatient eating disorders unit at the IRCCS Stella Maris Foundation. These adolescents (all between 11 and 18 years) were characterized by restrictive behaviors and weight loss purely by dieting without binge eating or purging behaviors. They fulfilled the criteria for the diagnosis of AN-R according to the Diagnostic and Statistical Manual of Mental Disorders, 4th Edition (DSM-IV). ${ }^{23}$ Patients with psychotic symptoms, intelligence quotient [IQ] below 80, medical pathology not correlated with the eating disorder, current or history of substance abuse, and significant intrinsic instability requiring constant medical care supervision (such as severe bradycardia, dehydration, or electrolyte imbalance) were excluded. The mean body mass index was $15.07 \pm 1.54 \mathrm{~kg} / \mathrm{m}^{2}$ and the mean duration of illness was $19.56 \pm 18.38$ months. Twenty-seven patients (84.38\%) fulfilled the criteria for an Axis I anxiety and/or mood disorder (assessed through the Italian version of the Kiddie-Sads-Present and Lifetime Version [K-SADS-PL] $)^{24}$ and 3 of these 27 patients also met the criteria for an Axis II Obsessive-Compulsive Personality Disorder (evaluated by the Structured Clinical Interview for DSM-IV Axis II Personality Disorders). ${ }^{25}$ Twelve patients (37.5\%) received psychopharmacological treatment with selective serotonin reuptake inhibitors and/or atypical antipsychotics and/or mood modulators, while the remaining 20 subjects were medication-naïve.

The AN-R clinical sample was compared to a healthy control group (HC) composed of 41 healthy female adolescents recruited from students (mean age $=14.02 \pm 1.69$ years; range $=11.08-17.16$ years) of a middle and a high school in the metropolitan area of Pisa. All control subjects attended regular classes and schools without a support teacher.

\section{Measures}

The following established measures, which were part of a larger suite of tests, were administered for this study.

\section{Assessment of empathic abilities}

The Italian version ${ }^{26}$ of the IRI ${ }^{4}$ was used to evaluate empathic abilities. The IRI is a 28 -item self-reported questionnaire that allows a multi-dimensional assessment of empathy, which can 
be measured by two cognitive subscales (perspective taking [PT]; fantasy [FS]) and two affective subscales (empathic concern [EC]; personal distress [PD]). Participants respond to each item using a 5-point Likert scale ranging from -2 "does not describe me well" to +2 "does describe me well". Therefore the scores of each subscale range between -14 and +14 points where higher scores indicate more empathic abilities.

\section{Assessment of eating disorder severity}

To address eating disorder severity, the following measures were considered: 1) the Body Mass Index (BMI) calculated by dividing body weight in kilograms by height in meters squared $\left.\left(\mathrm{kg} / \mathrm{m}^{2}\right) ; 2\right)$ the Eating Attitudes Test (EAT-26), ${ }^{27}$ a 26-item self-reported questionnaire of disordered eating patterns. The total score ranges from 0 to 78; a higher score reflects a greater degree of eating pathology, with a score over 20 indicating a possible eating problem; 3) disease duration, defined as the time interval between the onset of the first eating disorder symptoms and the administration of the tests.

\section{Assessment of possible psychopathological traits associated to the eating disorder}

To address psychopathological traits associated with the eating disorder, two questionnaires were used: 1) the Child Behavior Checklist (CBCL 6-18), ${ }^{28}$ a 118 item parentreported measure designed to record the problem behaviors as well as the competencies of children or adolescents. Each item describes a specific behavior and the parent is asked to rate its frequency on a 3-point Likert scale. The scoring gives a summary profile including internalizing (INT), externalizing (EXT), and total problems (TP), and a syndrome profile (withdrawn, anxious/depressed, somatic complaints, social problems, thought problems, attention problems, delinquent behavior, and aggressive behavior); 2) the Youth Self Report (YSR 11-18), an118 items self-reported questionnaire for adolescents from 11 to 18 years old. ${ }^{28}$ The YSR provides a summary profile and a syndrome profile paralleling those of the CBCL 6-18.

\section{Procedure}

The study was carried out according to the standards for good ethical practice of the IRCCS Stella Maris Foundation, and was approved by the educational board of each school of the HC group. Written informed consent from a parent or guardian of each patient was obtained. Patients and their parents completed all the tests (IRI, EAT-26, CBCL 6-18, and
YSR 11-18) during the first days of the clinical assessment. $\mathrm{HC}$, in addition to the IRI, completed the EAT-26 and the YSR 11-18, in order to rule out subjects with eating problems and/or Axis I psychopathology, respectively. In fact, the YSR has been found to show a significant correlation with clinical DSM-IV diagnoses. ${ }^{28-30}$ Thus, subjects with an EAT-26 score $>20$, and/or a score $>60$ on INT and/or EXT and/or TP at the YSR, were excluded from the study. $\mathrm{HC}$ data were collected by a physician resident in Child and Adolescent Psychiatry (PF) during school hours and informed consent was obtained from participants and their parents through the head teacher's office at each school.

\section{Data analysis}

A Student's $t$-test was used to compare differences in EAT-26, YSR, and IRI mean scores between AN-R and HC groups; multivariate analysis of variance (ANOVA) with EAT-26 and with INT, EXT, and TP mean scores of the YSR as a covariate was used to identify IRI scales discriminating between AN-R and HC groups; Spearman correlations were adopted to assess the relationship between IRI scales and 1) associated psychopathology (CBCL), and 2) eating disorder severity (BMI and disease duration), respectively.

$P<0.05$ was considered statistically significant. All statistical analyses were performed using SPSS 18.0 for Windows (IBM Corporation, Armonk, NY, USA).

\section{Results}

Independent $t$-test analysis indicated no significant differences between the AN-R and the HC groups in age $(t[71]=1.88 ; P=0.065)$. The two groups showed significant differences between EAT-26 $(t[70]=8.47 ; P<0.001)$ and YSR (INT: $t[71]=4.96 ; P<0.001$; EXT: $t[71]=2.68 ; P=0.009$; TP $t[71]=3.78 ; P<0.001)$ scores .

\section{Cognitive and affective empathy}

To examine the cognitive versus affective aspects of empathy, the total scores for the cognitive (PT + FS) scales and the affective (EC + PD) scales were calculated. As observed in Figure 1, AN-R had a significantly lower CE mean score than HC (AN-R mean score: $0.44 \pm 6.87$; HC mean score: $5.24 \pm 6.45 ; t[71]=3.0 ; P=0.003)$. The AN-R group scored significantly lower on both PT $(t[71]=2.36$; $P=0.021)$ and FS $(t[71]=2.18 ; P=0.032)$ scales. The two groups didn't show significant differences on AE mean score (AN-R mean score: $4.69 \pm 7.08$; HC mean score: $4.20 \pm 4.75 ; t[71]=0.35 ; P=0.725)$ and the two related subscales (see Table 1). 


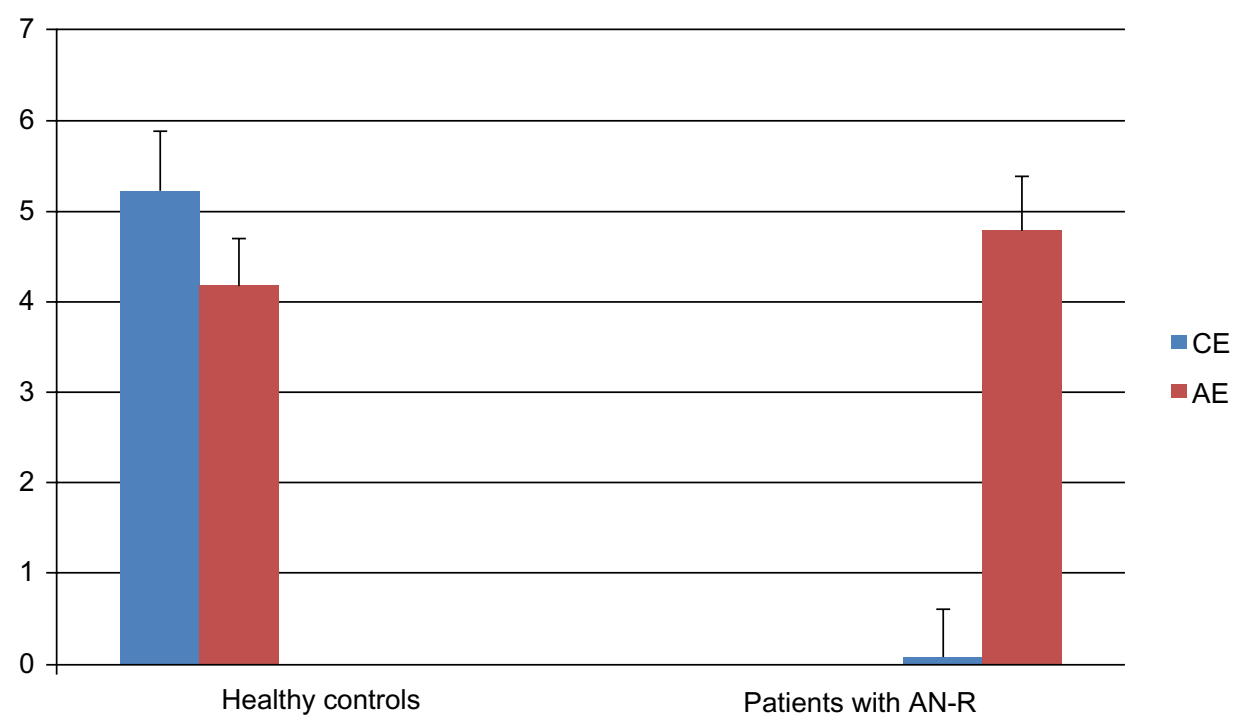

Figure I Mean score of cognitive empathy and affective empathy reported by healthy controls and patients with AN-R.

Notes: Cognitive empathy scores differed significantly between groups $(P<0.0 \mathrm{I})$, whereas no differences were found for affective empathy scores. Error bars represent standard error of the mean.

Abbreviations: $\mathrm{AE}$, affective empathy; $\mathrm{CE}$, cognitive empathy; $\mathrm{AN}-\mathrm{R}$, anorexia nervosa-restricting type.

\section{Cognitive empathy, disease severity, and associated psychopathology}

Significant differences between groups with respect to CE $(F[69]=5.19 ; P=0.008)$ and the PT subscale $(F[69]=3.70$; $P=0.03)$ when EAT-26 mean scores were used as a covariate; no significant differences on the FS subscale $(F[69]=2.61$; $P=0.081$ ) were obtained.

Significant differences between groups in IRI when YSR mean scores were used as a covariate were noted. Specifically, when INT was used as a covariate: $\mathrm{CE}(F[70]=5.15 ; P=0.008)$, $\mathrm{PT}(F[70]=2.41 ; P=0.098), \mathrm{FS}(F[70]=3.52 ; P=0.035)$; when EXT was used as a covariate: $\mathrm{CE}(F[70]=5.08 ; P=0.009)$, $\mathrm{PT}$ $(F[70]=5.24 ; P=0.008)$, FS $(F[70]=2.29 ; P=0.109)$; when TP was used as a covariate: $\mathrm{CE}(F[70]=4.46 ; P=0.015)$, PT $(F[70]=2.92 ; P=0.061)$, FS $(F[70]=3.25 ; P=0.045)$.

Table I Mean score (and standard deviation) of the IRI scales and group comparison

\begin{tabular}{|c|c|c|c|c|}
\hline \multirow[t]{2}{*}{ IRI scales } & \multicolumn{2}{|c|}{ Group means (SD) } & \multicolumn{2}{|c|}{$\begin{array}{l}\text { Group } \\
\text { comparison }\end{array}$} \\
\hline & $H C(n=4 I)$ & AN-R $(n=32)$ & $t$ & $P$ \\
\hline Cognitive empathy & $5.24(6.45)$ & $0.44(6.87)$ & 3.07 & $0.003 * *$ \\
\hline Perspective taking & $2.61(3.58)$ & $0.53(3.93)$ & 2.36 & $0.021 *$ \\
\hline Fantasy & $2.63(5.03)$ & $-0.09(5.62)$ & 2.18 & $0.033^{*}$ \\
\hline Affective empathy & $4.20(4.75)$ & $4.69(7.08)$ & 0.35 & 0.725 \\
\hline Empathic concern & $6.15(3.24)$ & $5.22(3.37)$ & 1.20 & 0.236 \\
\hline Personal distress & $-1.95(3.5 \mathrm{I})$ & $-0.53(5.56)$ & 1.33 & 0.187 \\
\hline
\end{tabular}

Notes: $* P<0.05 ; * * P<0.0$ I.

Abbreviations: IRI, Interpersonal Reactivity Index; AN-R, anorexia nervosarestricting type; $\mathrm{HC}$, healthy controls; SD, standard deviation.
No statistically significant associations between cognitive empathy and: 1) BMI (rho=-0.32; $P=0.069) ; 2$ ) disease duration $(r h o=-0.07 ; P=0.69)$; or 3) CBCL 6-18 (INT: $r h o=0.23$; $P=0.213 ; \mathrm{EXT}=-0.05 ; P=0.806 ; \mathrm{TP}=0.16 ; P=0.401)$ were detected.

\section{Discussion}

This study adds to the limited literature regarding empathy in AN-R and the results show statistically significant differences between patients with AN-R and the $\mathrm{HC}$ with respect to empathic profile assessed using the IRI. ${ }^{4}$ The main finding arising from the current investigation, performed on a carefully selected population of adolescent females with AN-R, is the impairment in empathic abilities limited to the cognitive domain, while sparing the affective component. Cognitive capacity is the ability to take the perspective of the other person in real (perspective taking scale) and in fictional (fantasy scale) situations. In particular, a low score on the PT scale is indicative of an impairment in interpersonal functioning, ${ }^{4}$ and converges with the repeated findings of altered social skills in anorectic patients. ${ }^{6}$ Moreover, since executive functions contribute to the effective development of $\mathrm{CE}^{31}$ and, at the same time, are consistently reported as impaired in AN subjects, ${ }^{32}$ further research is warranted to clarify the possibility that executive functions deficits interfere with the reduced $\mathrm{CE}$ abilities of $\mathrm{AN}$ patients.

Only two other studies assessed empathic abilities in AN-R through the IRI. ${ }^{33,34}$ In contrast with our results, these investigations reported no significant differences between 
patients and controls ${ }^{33}$ and higher ratings for emotional empathy in AN subjects than HC participants. ${ }^{34}$ Moreover, a mixed sample of both AN-R and AN-BE patients were enrolled in this latter study, whereas in the current investigation subjects who engaged in binging/purging behaviors were excluded. Hence, it is difficult to compare our work with the other two, as they do present substantial differences not only with respect to sample age (adolescents in our report versus adults in Guttman and Laporte, and Beadle et al $)^{33,34}$ and clinical severity (inpatients versus outpatients), but also as to the methods of score attribution. A lower $\mathrm{CE}$ and similar AE scores, compared to controls, is instead a finding that our AN patients have commonalities with Asperger subjects, ${ }^{17}$ providing further support for the similarities between the two disorders. ${ }^{7,8}$

Results point out specific CE impairment in adolescents with AN-R. In particular, this finding suggests three key issues: first, on the basis of its independence from associated psychopathology assessed through the YSR, a $\mathrm{CE}$ deficit could be considered a specific marker of the adolescent AN-R, not mediated by comorbid symptoms; second, the independence of a $\mathrm{CE}$ deficit from the severity of illness suggests that it is not directly attributable to the profound effects of starvation on neuropsychological functioning. ${ }^{35}$ In the future, longitudinal studies comparing ill and recovered AN-R patients may clarify whether the reduction in $\mathrm{CE}$ is a state-correlated characteristic or if it also persists after recovery. Third, the absence of correlation between $\mathrm{CE}$ scores and disease duration indicates the possible presence of a deficit in $\mathrm{CE}$ from the beginning of the disorder or even before AN-R onset. The hypothesis of CE impairment as a precursor of AN-R is consistent with other studies on the personality traits involved in creating a predisposition to developing this disorder. ${ }^{1,36}$ In this view, low $\mathrm{CE}$ could become one of the risk factors that precede the onset of a restrictive eating disorder. However, a prospective exploration of empathic skills in a non-clinical population is required to sustain the hypothesis of an association with the subsequent development of AN.

Finally, empathic impairment limited to the cognitive domain supports its functional independence from the affective one; neuroimaging (for a review, see Decety and Meyer $)^{37}$ as well as lesion studies ${ }^{5}$ sustain this hypothesis. In particular, two distinct brain systems for cognitive and $\mathrm{AE}$ have been described: the ventromedial prefrontal cortex is involved in CE, whereas the inferior frontal gyrus is a part of the AE network. Interestingly, ventromedial prefrontal cortex anomalies have been frequently reported in neuroimaging studies conducted in AN patients (eg, Uher et al); ${ }^{38,39}$ future functional MRI research is needed to investigate the response to empathy-eliciting stimuli in AN-R.

\section{Conclusion}

The main result of this study is that adolescents with AN-R type do not show global empathy abilities deficit, but rather a specific CE impairment. The current findings need to be qualified by a number of limitations. Specifically, self-reported measurements' lack of objectivity, can be biased by denial or exaggeration of symptoms, as well as social desirability, and only reflect the self-perception of empathic characteristics. Moreover, a subject may not understand a question and give a misleading answer. However, this issue was mitigated in the present study by having a clinical psychologist remain with the subject in order to clarify possible misunderstandings during the administration of the IRI scale.

Secondly, the control subjects were screened for possible Axis I psychopathology through the YSR. However, besides the possible false positive and false negative results, the YSR does not evaluate personality disorders that could be present in the subjects enrolled in the study as controls.

The vast majority (84.4\%) of AN-R patients involved in this study also meet diagnostic criteria for an additional internalizing diagnosis assessed through the K-SADS-PL. ${ }^{24}$ Since internalizing disorders could impact empathic abilities, ${ }^{19}$ we cannot rule out that the low levels of CE may be due to the influence of comorbid psychiatric symptoms rather than to AN-R itself. Also, while subjects with major depressive disorders assessed through the IRI reported significantly lower levels of both cognitive and affective empathy, ${ }^{19}$ our AN-R patients showed a selective impairment of CE. However, in order to investigate the specific role of internalizing symptoms on empathic abilities, future studies should include an additional control sample composed of depressed/anxious subjects matched for demographic characteristics.

Further, the selective inclusion criteria of the patient group imply a relatively small sample size that limits the statistical power of results. Notwithstanding the problematic issues raised above, these data, if supported by a larger replication study, could have a 3-fold implications for AN-R: a) clinical, since the overall management of these patients should take into account the compromised empathic skills; b) therapeutic, with the development of specific intervention goals focused on CE enhancement; and c) preventive, with the aim of early individuation of subjects with CE deficits, a marker that, in addition to other susceptibility factors, may 
play an important role in the development and maintenance of the anorexic disorder.

\section{Acknowledgments}

The authors thank the adolescents and parents who participated in the study.

\section{Disclosure}

The authors report no conflicts of interest in this work.

\section{References}

1. Wentz E, Gillberg IC, Anckarsäter H, Gillberg C, Råstam M. Adolescent-onset anorexia nervosa: 18-year outcome. Br J Psychiatry. 2009;194(2):168-174.

2. Diagnostic and Statistical Manual of Mental Disorders. 5th ed. Washington, DC: American Psychiatric Association; 2013.

3. Godart NT, Perdereau F, Curt F, et al. Predictive factors of social disability in anorexic and bulimic patients. Eat Weight Disord. 2004;9(4):249-257.

4. Davis MH. Measuring individual differences in empathy: Evidence for a multidimensional approach. J Pers Soc Psychol. 1983;44(1): $113-126$.

5. Shamay-Tsoory SG, Aharon-Peretz J, Perry D. Two systems for empathy: a double dissociation between emotional and cognitive empathy in inferior frontal gyrus versus ventromedial prefrontal lesions. Brain. 2009;132(Pt 3):617-627.

6. Gillberg IC, Råstam M, Gillberg C. Anorexia nervosa 6 years after onset: Part I. Personality disorders. Compr Psychiatry. 1995;36(1):61-69.

7. Anckarsäter H, Hofvander B, Billstedt E, et al. The sociocommunicative deficit subgroup in anorexia nervosa: autism spectrum disorders and neurocognition in a community-based, longitudinal study. Psychol Med. 2012;42(9):1957-1967.

8. Oldershaw A, Treasure J, Hambrook D, Tchanturia K, Schmidt U. Is anorexia nervosa a version of autism spectrum disorders? Eur Eat Disord Rev. 2011;19(6):462-474.

9. Hambrook D, Tchanturia K, Schmidt U, Russell T, Treasure J. Empathy, systemizing, and autistic traits in anorexia nervosa: a pilot study. $\mathrm{Br} J$ Clin Psychol. 2008;47(Pt 3):335-339.

10. Tchanturia K, Happé F, Godley J, et al. "Theory of Mind" in anorexia nervosa. Eur Eat Disord Rev. 2004;12:361-366.

11. Russell TA, Schmidt U, Doherty L, Young V, Tchanturia K. Aspects of social cognition in anorexia nervosa: affective and cognitive theory of mind. Psychiatry Res. 2009;168(3):181-185.

12. Oldershaw A, Hambrook D, Tchanturia K, Treasure J, Schmidt U. Emotional theory of mind and emotional awareness in recovered anorexia nervosa patients. Psychosom Med. 2010;72(1):73-79.

13. Medina-Pradas C, Blas Navarro J, Álvarez-Moya EM, et al. Emotional theory of mind in eating disorders. Int $J$ Clin Health Psychol. 2012;12(2):189-202.

14. Calderoni S, Muratori F, Leggero C, et al. Neuropsychological functioning in children and adolescents with restrictive-type anorexia nervosa: an in-depth investigation with NEPSY-II. J Clin Exp Neuropsychol. 2013;35(2):167-179.

15. Shamay-Tsoory SG, Harari H, Aharon-Peretz J, Levkovitz Y. The role of the orbitofrontal cortex in affective theory of mind deficits in criminal offenders with psychopathic tendencies. Cortex. 2010;46(5): 668-677.

16. Harari H, Shamay-Tsoory SG, Ravid M, Levkovitz Y. Double dissociation between cognitive and affective empathy in borderline personality disorder. Psychiatry Res. 2010;175(3):277-279.

17. Rogers K, Dziobek I, Hassenstab J, Wolf OT, Convit A. Who cares? Revisiting empathy in Asperger syndrome. J Autism Dev Disord. 2007;37(4):709-715.
18. Shamay-Tsoory SG, Shur S, Barcai-Goodman L, Medlovich S, Harari H, Levkovitz Y. Dissociation of cognitive from affective components of theory of mind in schizophrenia. Psychiatry Res. 2007;149(1-3): $11-23$.

19. Cusi AM, Macqueen GM, Spreng RN, McKinnon MC. Altered empathic responding in major depressive disorder: relation to symptom severity, illness burden, and psychosocial outcome. Psychiatry Res. 2011;188(2): 231-236.

20. Shamay-Tsoory S, Harari H, Szepsenwol O, Levkovitz Y. Neuropsychological evidence of impaired cognitive empathy in euthymic bipolar disorder. J Neuropsychiatry Clin Neurosci. 2009;21(1): 59-67.

21. Maurage P, Grynberg D, Noël X, et al. Dissociation between affective and cognitive empathy in alcoholism: a specific deficit for the emotional dimension. Alcohol Clin Exp Res. 2011;35(9):1662-1668.

22. Godart NT, Perdereau F, Rein Z, et al. Comorbidity studies of eating disorders and mood disorders. Critical review of the literature. $J$ Affect Disord. 2007;97(1-3):37-49.

23. Diagnostic and Statistical Manual of Mental Disorders. 4th ed, text revision. Washington: American Psychiatric Association; 2000.

24. Kaufman J, Birmaher B, Brent D, et al. Schedule for Affective Disorders and Schizophrenia for School-Age Children-Present and Lifetime Version (K-SADS-PL): initial reliability and validity data. $J$ Am Acad Child Adolesc Psychiatry. 1997;36(7):980-988.

25. First MB, Gibbon M, Spitzer RL, et al. Structured Clinical Interview for DSM-IV Axis II Personality Disorders (SCID-II). Washington; American Psychiatric Press: 1997.

26. Albiero P, Ingoglia S, Lo Coco A. Contributo all'adattamento italiano dell'Interpersonal Reactivity Index. [Contribution to the Italian adaptation of the Interpersonal Reactivity Index]. Testing Psicometria Metodologia. 2006;13(2):107-125. Italian.

27. Garner DM, Olmsted MP, Bohr Y, Garfinkel PE. The eating attitudes test: psychometric features and clinical correlates. Psychol Med. 1982;12(4):871-878.

28. Achenbach TM, Rescorla LA. Manual for the ASEBA School-Age forms and Profiles. Burlington: University of Vermont, Research Center for Children, Youth, and Families; 2001.

29. Aebi M, Metzke CW, Steinhausen HC. Prediction of major affective disorders in adolescents by self-report measures. J Affect Disord. 2009;115(1-2):140-149.

30. Ferdinand RF. Validity of the CBCL/YSR DSM-IV scales Anxiety Problems and Affective Problems. J Anxiety Disord. 2008;22(1): 126-134.

31. Decety J, Jackson PL. The functional architecture of human empathy. Behav Cogn Neurosci Rev. 2004;3(2):71-100.

32. Jáuregui-Lobera I. Neuropsychology of eating disorders: 1995-2012. Neuropsychiatr Dis Treat. 2013;9:415-430.

33. Guttman HA, Laporte L. Empathy in families of women with borderline personality disorder, anorexia nervosa, and a control group. Fam Process. 2000;39(3):345-358.

34. Beadle JN, Paradiso S, Salerno A, McCormick LM. Alexithymia, emotional empathy, and self-regulation in anorexia nervosa. Ann Clin Psychiatry. 2013;25(2):107-120.

35. Keys A, Brozek J, Henschel A, et al. The biology of human starvation. Minneapolis: Minnesota University Press; 1950.

36. Rothschild-Yakar L, Levy-Shiff R, Fridman-Balaban R, Gur E, Stein D. Mentalization and relationships with parents as predictors of eating disordered behavior. J Nerv Ment Dis. 2010;198(7):501-507.

37. Decety J, Meyer M. From emotion resonance to empathic understanding: a social developmental neuroscience account. Dev Psychopathol. 2008;20(4):1053-1080

38. Uher R, Brammer MJ, Murphy T, et al. Recovery and chronicity in anorexia nervosa: brain activity associated with differential outcomes. Biol Psychiatry. 2003;54(9):934-942.

39. Uher R, Murphy T, Brammer MJ, et al. Medial prefrontal cortex activity associated with symptom provocation in eating disorders. Am J Psychiatry. 2004;161(7):1238-1246. 
Neuropsychiatric Disease and Treatment

Dovepress

\section{Publish your work in this journal}

Neuropsychiatric Disease and Treatment is an international, peerreviewed journal of clinical therapeutics and pharmacology focusing on concise rapid reporting of clinical or pre-clinical studies on a range of neuropsychiatric and neurological disorders. This journa is indexed on PubMed Central, the 'PsycINFO' database and CAS.

The manuscript management system is completely online and includes a very quick and fair peer-review system, which is all easy to use. Visit http://www.dovepress.com/testimonials.php to read real quotes from published authors.

\footnotetext{
Submit your manuscript here: http://www.dovepress.com/neuropsychiatric-disease-and-treatment-journal
} 